\title{
ALKAPTONURIA DIAGNOSED IN A GERIATRIC PATIENT; A CASE REPORT
}

Turkish Journal of Geriatrics DOI: 10.31086/tigeri.2020.148 2020; 23(1): 147-152

- Ramazan GÜNDÜZ1 1 D - Betül ÜSTÜN 1 (D) - Ayça URAN ŞAN 11 D - Hatice Gülşah KARATAŞ ${ }^{1}$ (D) - Müfit AKYÜZ1 (D)

CORRESPONDANCE

\section{Ayça URAN ŞAN}

Karabuk University Research and Training Hospital, Department of Physical Therapy and Rehabilitation Medicine, Karabuk, TURKEY.

Phone: +905362088978

e-mail: dr.uran@hotmail.com

Received: 17/02/2020

Accepted: 05/03/2020

${ }^{1}$ Karabuk University Research and Training Hospital, Department of Physical Therapy and Rehabilitation Medicine, Karabuk, TURKEY.

\section{A}

Alkaptonuria is a hereditary and metabolic disease which is characterised by ocranosis, arthritis and aciduria. It is a rare clinical condition which has an estimated incidence varies from $1 / 250,000$ to $1 / 1000000$ live births. This autosomal recessive disorder occurs as a result of homogentisic acid dioxygenase enzyme deficiency which takes part in tyrosine metabolism. This case report aimed to present a geriatric patient who had diagnosed alkaptonuria. A 69 years old male was admitted to our department with knee-shoulder pain and range of motion (ROM) limitation. His medical history revealed that he had both mechanical and inflammatory joint pain for 22 years. The examination of his eyes were compatible with blue sclera and there was also bluish black discoloration on the ears. He had also hearing loss. Qualitative 24 hours collected urine examination showed dark black discoloration. The patient was diagnosed as alkaptonuria. A physical therapy programme were planned to the patient by our department. Clinicians should be aware of this pathology and the diagnosis should be confirmed by clinical, laboratory and radiological examinations.

Keywords: Alkaptonuria; Pain; Arthritis. 


\section{INTRODUCTION}

Alkaptonuria is a hereditary and metabolic disease that is characterized by ochronosis, arthritis, and aciduria (1). The disease was described by Garrod in 1902 (2). It is a rare clinical condition with an estimated incidence that varies from 1/250,000 to $1 / 1,000,000$ live births(3). This autosomal recessive disorder occurs as a result of the deficiency of homogentisic acid (HGA) dioxygenase enzyme, which takes part in tyrosine metabolism. The unexcreted HGA accumulates in various parts of the body, such as the skin, optic compartments, cardiovascular structures, and connective tissues of the musculoskeletal system with a bluish-black discoloration phenomenon termed as "ochronosis" (4). The diagnosis of alkaptonuriais based on clinical findings and urinary tests (5). Urine sample analysis that exhibits the presence of HGA is the gold standard for the confirmation of its diagnosis (5). Further, 24-hour urine collection and chromatographic techniques can be useful (5). Additionally, genetic tests could determine if an individualis homozygous or compound heterozygous (5). Although there is no treatment that could improve alkaptonuria, several studies reveal that nitisinone, which inhibits the tyrosine catabolic pathway, and dietary restriction of tyrosine and phenylalanine could be used as treatment options $(6,7)$. Here we present the case of a geriatric patient who was diagnosed with alkaptonuria.

\section{CASE REPORT}

A 69-year-old man was admitted to our department with knee and shoulder pain and range of motion (ROM) limitation. His medical history revealed that he had both mechanical and inflammatory knee and shoulder joint pain for 22 years. The level of his pain had been increased by moving and had a tendency to worsen at nights. He underwent total hip replacement operations bilaterally as a result of falls 6 and 14 years ago. His walking distance had decreased owing to the knee pain, and he could walk with a staff. The level of his pain was $100 \mathrm{~mm}$ based on a visual analog scale (VAS) from 0 to $100 \mathrm{~mm}$. His pain was significant and caused functional impairments in his daily activities. Upon physical examination, ROM limitation on both knee and shoulder joints was detected. Moreover, effusion was detected on the knees bilaterally. He felt pain during the ROM examination. Upon examination of his eyes, it was compatible with a blue sclera, and there was a bluish-black discoloration on his ears. Laboratory findings revealed that serum C-reactive protein (CRP) level was 156.47 $\mathrm{mg} / \mathrm{L}$, erythrocyte sedimentation rate (ESR) was $93 \mathrm{~mm} / \mathrm{h}$, white blood cell count (WBC) was 6.97 $\times 109 / L$, and rheumatologic tests were negative. Urine examination showed multiple WBCs with a negative urine culture. Qualitative 24-hour-collected urine examination showed dark black discoloration. Symmetrical joint space loss, chondrocalcinosis and subchondral sclerosis were detected in both knee and shoulder X-ray graphics (Figures 1 and 2). Furthermore, there were multilevel intervertebral disk calcifications, disk space narrowing, and syndesmophytes in cervicothoracolumbar graphics (Figure 3). He was consulted in the cardiology department owing to the complaint of dyspnea. The degeneration in his aortic valve was detected by echocardiography. On the basis of the clinical, laboratory, and radiological findings, he was diagnosed with alkaptonuria presenting with ochronosis. A physical therapy program, including cold pack, transcutaneous electrical nerve stimulation, ROM, stretching, and progressive resistance exercises was planned for the patient by our department. In addition, he was treated with $50 \mathrm{mg}$ diclofenac sodium (3 tablets/day) and 0.5 mg colchicum dyspert (2 tablets/day). After the physical therapy program, his VAS pain score decreased from 100 to $60 \mathrm{~mm}$. The treatment helped to ameliorate his swelling. Furthermore, the serum levels of his CRP and ESR had decreased after the treatment. 
Figure 1. Knee radiograph of knees shows narrowing in joint spaces, subchondral calcifications and degenerative changes of articular surfaces.

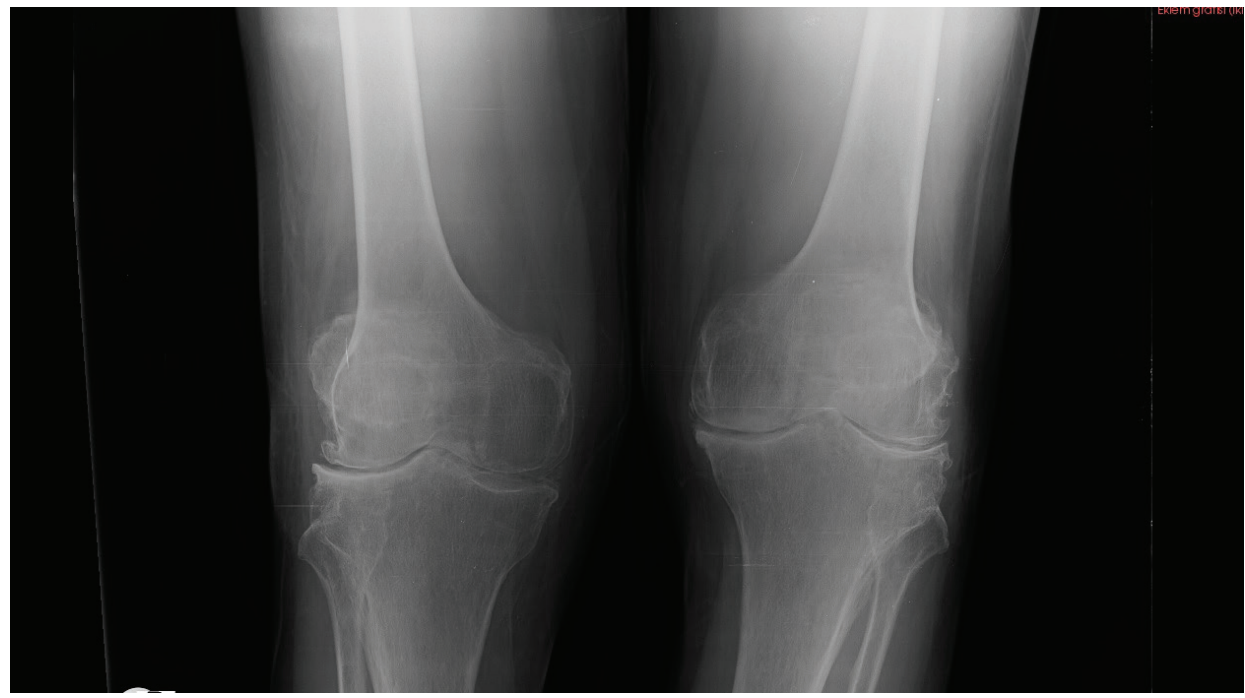

Figure 2. X-ray of shoulder shows significant calcifications, degenerative changes and narrowing of glenohumeral joint space.

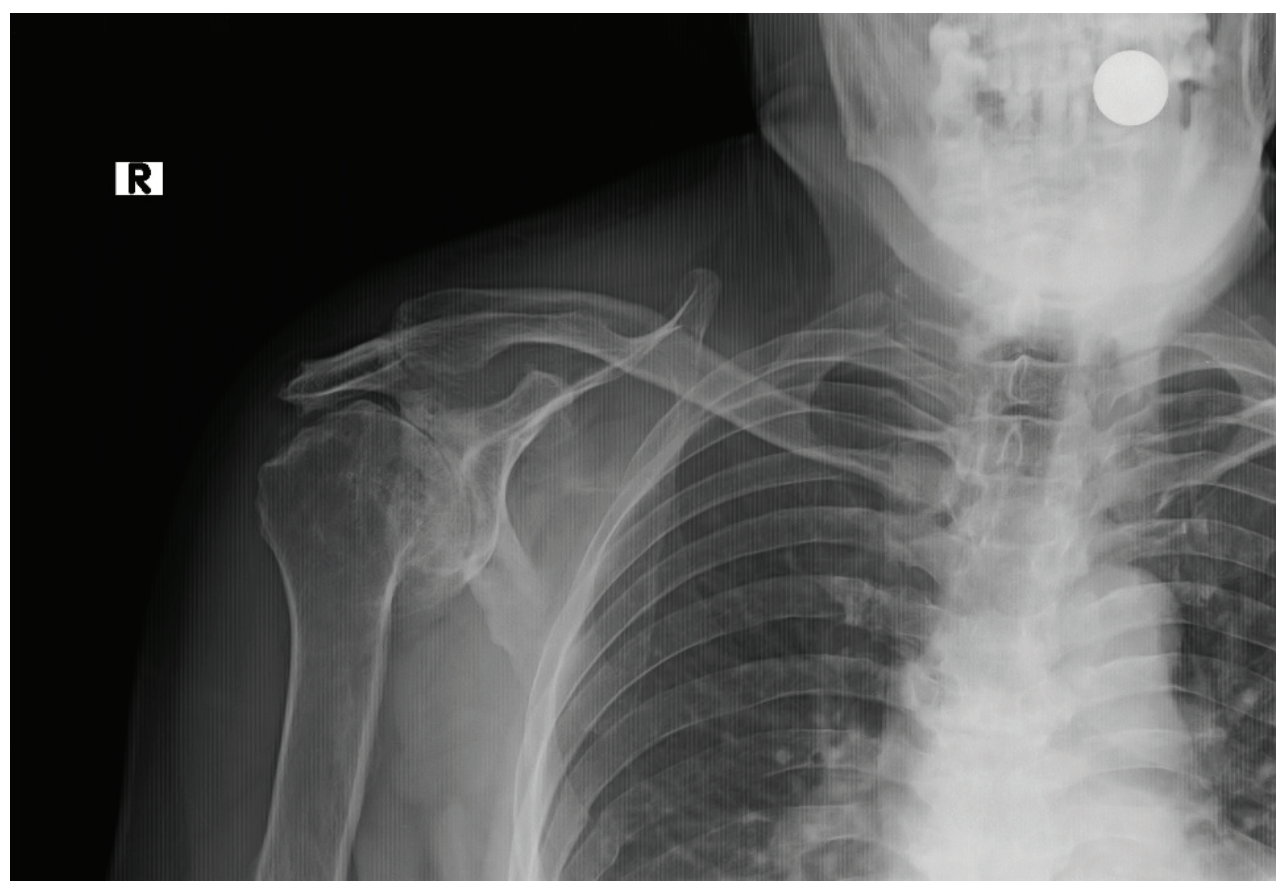


Figure 3. Radiograph of lumbar vertebral column shows narrowing in disc spaces and severe calcifications of intervertebral discs.

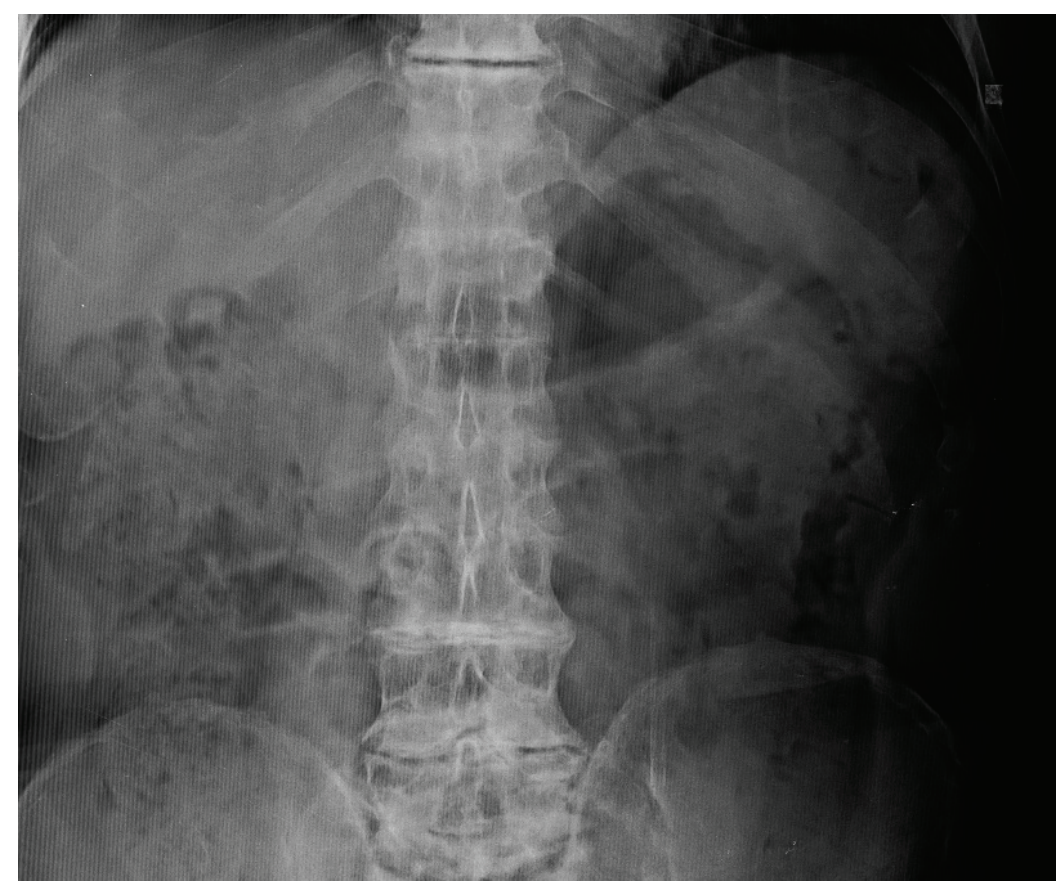

\section{DISCUSSION}

Alkaptonuria is a rare disorder of aromatic amino acid metabolism that has a Mendelian recessive inheritance pattern (1). The intracellular and extracellular accumulation of HGA, which plays an important role in phenylalanine and tyrosine metabolism, leads to this disorder (8). HGA is converted to maleylacetoacetic acid (MAA) by HGA dioxygenase enzyme in healthy individuals (5). The mutation in chromosome 3q21-q23 has been identified in alkaptonuria $(6,7)$. In this group of mutated patients, the conversion of HGA to MAA cannot be completed; therefore, HGA accumulates in various parts of the body and is excreted in the urine (5). As a result, HGA converts to benzoquinone acetate by oxidation and benzoquinone acetate monomers combine to generate melanin-like polymers in the tissues (8). Because of the polymerization and oxidation reactions of HGA, the color of the urine may turn into black or dark brown (9). Additionally, the polymerized HGA forms bluish-black discoloration called "ochronosis" that could exist in different parts of the body, such as the ear, sclera (Osler sign), joint capsules, cartilage, tendons, and ligaments $(10,11)$. Repeated dark brown or black discoloration of the urine during childhood is an important clue for the diagnosis; however, the prominent perspicuity of alkaptonuria in adults is a sign of arthritis (12). The symptoms of arthritis generally present in the third decade of life and ochronosis is formed after 30 years of age (13). Nevertheless, it is possible to diagnose the disease during infancy because of the darkening of the urine (12). To the best of our knowledge, 
our patient is one of the oldest cases diagnosed with alkaptonuria in the literature. In this study, we reported the case of a 69-year-old patient with alkaptonuria who was previously undiagnosed. $\mathrm{He}$ had been living in a rural area and stated that he had rarely preferred to take medical consultation owing to the long distance to the hospital. We believe that this condition plays an important role in the delay of the diagnosis.

Alkaptonuric ochronosis can lead to more brittle cartilages and eventually, fractures may arise (11). Our patient had a history of total hip replacement operations bilaterally owing to acetabular and femoral neck fractures. Based on the studies in the literature, total joint replacement treatment in patients with ochronosis has successful results, similar to that in patients with osteoarthritis $(14$, 15).

The spinal involvement includes spondylosis, disc herniation, and vertebral osteophyte formations that conduce pain in the patients (11). It is possible to determine the evidence of the disease by radiographic examination (11). X-ray findings comprise calcifications, narrowing of joint spaces, bony spurs, ankylosis and kyphosis $(9,11)$.

Furthermore, ochronosis leads to the accumulation of HGA pigments in the sclera, cartilage of the ears, and dermis of the skin, which becomes blue-black in color (12). Renal stones, prostate pathologies, and coronary artery calcifications may

\section{REFERENCES}

1. Reddy OJ, Gafoor JA, Suresh B, Prasad PO. Alkaptonuriawith review of literature. JNTRUniv Health Sci 2014;3:125-9. [Internet] Available from: http:// www.jdrntruhs.org/text.asp?2014/3/2/125/134884. Accessed:27.12.2019.

2. Garrod AE. The incidence of alkaptonuria: a study in chemical individuality 1902. Yale J Biol Med 2002;75(4): 221-31. (PMID: 12784973).

3. Phornphutkul C, Introne WJ, Perry MB, et al. Natural history of alkaptonuria. New England Journal of Medicine 2002;347(26):2111-21. (PMID:12501223). also accompany the clinical features of the disease (3).Further, aortic involvement was also observed in our patient. The diagnosis in our patient was made on the basis of the ochronotic pigmentation of the sclera and ear cartilage, arthritis, cardiac calcifications, radiological findings, and urine color that turns black upon standing.

Since, the complaint of pain in geriatric patients is often due to the arthrosis; it is important to make the differential diagnosis of this clinical condition. A detailed examination of the patient and the detection of clinical signs such as color discoloration of urine and the formation of ochronosis in various parts of the body will benefit the clinician for the diagnosis. Due to the fact that alkaptonuria is a systemic involvement disease; the determination of urinary and cardiac pathologies besides radiological findings will also be able to support the diagnosis in clinical practice.

In conclusion, alkaptonuriais a rare metabolic disorder that can be difficult to distinguish from other pathologies, especially in patients with older ages. Hence, clinicians should be aware of this pathology and the diagnosis should be confirmed by clinical, laboratory and radiological examinations.

\section{ACKNOWLEDGEMENT}

No funding was received in our study. All authors have no conflict of interest for this article.

4. Braconi D, Millucci L, Bernardini G, Santucci A. Oxidative stress and mechanisms of ochronosis in alkaptonuria. Free Radic Biol Med 2015;88:70-80. (PMID:25733348).

5. Ranganath LR, Jarvis JC, Gallagher JA. Recent advances in management of alkaptonuria. Journal of Clinical Pathology2013;66(5):367-73. (PMID:23486607).

6. Suwannarat $\mathrm{P}, \mathrm{O}^{\prime}$ Brien $\mathrm{K}$, Perry $\mathrm{MB}$, et al. Use of nitisinone in patients with alkaptonuria. Metabolism 2005;54(6):719-28. (PMID:15931605).

7. Wolff JA, Barshop B, Nyhan WL, et al. Effects of ascorbic acid in alkaptonuria: alterations in 
benzoquinone acetic acid and an ontogenic effect in infancy. Pediatr Res 1989;26(2):140-4. (PMID: 2771520).

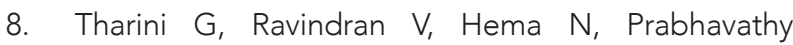
D, Parveen B. Alkaptonuria. Indian J Dermatol 2011;56(2):194-6. (PMID: 21716546)

9. Khatu SS, More YE, Vankawala D, Chavan D, Gökhale NR. Alkaptonuria: case report. Med J DY Patil Univ 2015;8:84-6. [Internet] Available from: http:// www.mjdrdypu.org/text.asp?2015/8/1/84/148860. Accessed:27.12.2019.

10. Thomas M,Jebaraj Jl, Thomas M, George R, et al. Acral pigmentation in alkaptonuria resembling degenerative collagenous plaques of the hands: a report of five cases. J Am Acad Dermatol 2011;65(2):45-6. (PMID: 21763547).

11. Albers SE, Brozena SJ, Glass LF, Fenske NA, et al. Alkaptonuria and ochronosis: case report and review. Journal of the American Academy of Dermatology 1992;27(4): 609-14. (PMID:1401313).

12. Thalagahage KN, Jayaweera JA, Kumbukgolla WW, Senavirathne I. Detection of alkaptonuria in a 1-weekold infant. BMJ Case Rep 2015 May 8; 2015:1-2. [Internet] Available from: http://www.ncbi.nlm.nih. gov/pmc/articles/PMC4434267/pdf/bcr-2014-208505. pdf. Accessed:27.12.2019.
13 Introne WJ, Gahl WA. Alkaptonuria, In: Adam MP, Ardinger $\mathrm{HH}$, Pagon RA, et al.(Eds) GeneReviews ${ }^{\circledR}$ University of Washington, Seattle;1993-2019. [Internet]. Available from: http://www.ncbi.nlm.nih. gov/books/NBK1454.Accessed:27.12.2019.

14. Franz A, Abusafieh M. Severe osteoarthritis of the knee as an early symptom of alkaptonuria: a case report. Shafa Ortho J 2016;3(1):45-7. [Internet] Available from:http://neoscriber.org/cdn/serve/e3/78/ e3784d52eb79e3f889a6785e98ec9ae3be16b095// soj-03-01-4547.pdf. Accessed:27.12.2019.

15. Ozmanevra R, Güran $O$,Karatosun V,Günal i, et al. Total knee arthroplasty in ochronosis: a case report and critical review of the literature. Joint Diseases \& Related Surgery 2013;24(3):169-72. (PMID: 24191883). 